\title{
Radiation Damping in FRW Space-times with Different Topologies
}

\author{
A. Bernut, G.I. Gomerol, M.J. Rebouças \\ Centro Brasileiro de Pesquisas Físicas \\ Departamento de Relatividade e Particulas \\ Rua Dr. Xavier Sigaud 150 \\ 22290-180 Rio de Janeiro - RJ, Brazil
}

(June 14, 2021)

\begin{abstract}
We study the role played by the compactness and the degree of connectedness in the time evolution of the energy of a radiating system in the Friedmann-Robertson-Walker (FRW) space-times whose $t=$ const spacelike sections are the Euclidean 3 -manifold $\mathcal{R}^{3}$ and six topologically non-equivalent flat orientable compact multiply connected Riemannian 3-manifolds. An exponential damping of the energy $E(t)$ is present in the $\mathcal{R}^{3}$ case, whereas for the six compact flat 3-spaces it is found basically the same pattern for the evolution of the energy, namely relative minima and maxima occurring at different times (depending on the degree of connectedness) followed by a growth of $E(t)$. Likely reasons for this divergent behavior of $E(t)$ in these compact flat 3-manifolds are discussed and further developments are indicated. A misinterpretation of Wolf's results regarding one of the six orientable compact flat 3-manifolds is also indicated and rectified.
\end{abstract}

PACS numbers: 98.80.-k, 98.80.Hw, 04.20.Gz, 04.20.Jb, 04.20.Cv

\section{INTRODUCTION}

As general relativity is a purely metrical (local) theory it clearly leaves unsettled the global structure (topology) of space-time. However, in cosmology perhaps the most important problems are related to the global structure of space-time, where the topological degrees of freedom ought to play an essential role.

Geometry constrains, but does not determine the topology of a space-time. Consider, for example, the FriedmannRobertson-Walker (FRW) space-times, whose line element can be given by

$$
d s^{2}=d t^{2}-A^{2}(t)\left[\frac{d r^{2}}{1-\kappa r^{2}}+r^{2}\left(d \theta^{2}+\sin ^{2} \theta d \varphi^{2}\right)\right],
$$

where $A(t)$ is the scale factor, $t$ is the cosmic time, and the constant spatial curvature $\kappa=0, \pm 1$ specifies the type of geometry (flat, elliptic or hyperbolic) of the $t=$ const spacelike section $\mathcal{M}_{3}$. Clearly FRW space-time manifolds $\mathcal{M}_{4}$ can be splitted into $\mathcal{R} \times \mathcal{M}_{3}$. The number of three-dimensional spacelike manifolds $\mathcal{M}_{3}$ which can be endowed with the three possible geometries of the $t=$ const 3 -spaces of FRW space-time is quite large 11.21: for $\kappa=0$ there are 18 topologically distinct 3-spaces, while for both $\kappa= \pm 1$ an infinite number of 3-spaces exist [2,3]. Even if we restrict ourselves to orientable and compact manifolds we still have an infinite number of 3 -spaces $\mathcal{M}_{3}$ for the elliptic and hyperbolic cases, and six families of topologically different spacelike manifolds for the flat 3-spaces [1] - [《].

Since physical laws are usually expressed in terms of local differential equations, in order to be confident about the physical results one derives it is often necessary to have some degree of control over the topological structure of the space-time manifold so as to include constraints imposed by the topology [5]. One is then confronted with the question of what topologies are physically acceptable for a given space-time geometry. An approach to this problem is to study the possible observational (or physical) consequences of adopting particular topologies for the space-time [6] - 19] (see also [3] and references therein).

In this work we study the role played by the compactness and the degree of connectedness in the time evolution of the energy of a radiating system in the flat FRW space-times whose spacelike $t=$ const sections are endowed with seven different topologies, namely the simply connected 3-space $\mathcal{R}^{3}$, and six multiply connected orientable compact 3-manifolds obtained by suitable identifications of opposite faces of cubes (four) and of hexagonal prisms (two) after suitable turns [2, 1,41 .

The radiating system we shall be concerned with is represented by a point-like harmonic oscillator (energy source) coupled with a relativistic massless scalar field [20] - 29]. Similar radiating systems have been used to study a wide class of radiation phenomena as, for example, gravitational waves [23] or the radiated energy of oscillating electromagnetic dipoles 30, 31. Our radiating system was also used in the study of dynamic and geometric constraints on the radiation in elliptic FRW expanding universes [27] - 29]. 
In the next section the radiating system is described: the action integral is presented, its variation is performed and the corresponding evolution equations are obtained. We also outline there a proposal for solving these equations. In section III the Green functions of the wave operator are derived for each specific space-time manifold with arbitrary scale factor $A(t)$, and combined with the evolution equations to give the corresponding radiation reaction equations. A misinterpretation [2] - [4] of Wolf's [1] results (Theorem 3.5.5) regarding one of the six orientable compact flat 3 -manifolds is also indicated and rectified therein. In section IV, the radiation reaction equations are numerically integrated. Graphs that show how the system energy varies with the time for each topologically different flat FRW manifold are presented for static and non-static cases. Our main conclusions are discussed in section $\mathrm{V}$. We show that when the $t=$ const sections are the simply connected 3 -space $\mathcal{R}^{3}$ the radiation damping phenomenon is present, whereas for all compact flat FRW space-time manifolds we have investigated, the energy $E(t)$ exhibits a few relative minima and maxima followed by a growth of the energy with the time. Possible reasons for this divergent behavior of $E(t)$ in these compact flat 3-manifolds are examined. We also discuss the role played by the compactness and by the degree of connectedness in the energy patterns of our system in these space-time manifolds. Further developments are indicated therein.

\section{PHYSICAL SYSTEM AND EVOLUTION EQUATIONS}

Radiation waves produced by an oscillating energy source as, e.g., an oscillating electromagnetic dipole, have been studied by using a theoretical model represented by a classical harmonic oscillator (energy source) coupled with a relativistic massless scalar field (scalar radiation waves propagating at speed of light) [20]- 28]. In our model the gravitational field is treated as external, but a suitable conformal coupling with the scalar field is considered [32].

The dynamics of our system can be described by an action integral, which contains a term for the scalar field $\phi(t, \vec{x})$, another associated to the oscillation amplitude $Q(t)$ of the point-like harmonic oscillator, and a coupling term between the scalar field and the harmonic oscillator according to

$$
\begin{aligned}
S & =\frac{1}{2} \int d^{4} x \sqrt{-g}\left[g^{\mu \nu} \partial_{\mu} \phi \partial_{\nu} \phi-\frac{1}{6} \hat{R} \phi^{2}\right]+\frac{1}{2} \int d t\left[\dot{Q}^{2}-\omega_{\varepsilon}^{2} Q^{2}\right] \\
& +\lambda \int d^{4} x \sqrt{-g} \rho(t, \vec{x}) Q(t) \phi(t, \vec{x})
\end{aligned}
$$

where $t \in\left[t_{0}, \infty\right), \vec{x} \in \mathcal{M}_{3}, g_{\mu \nu}$ is the metric tensor on $\mathcal{M}_{4}, g \equiv \operatorname{det}\left(g_{\mu \nu}\right), \hat{R}$ is the scalar curvature of $\mathcal{M}_{4}$, overdot means derivative with respect to $t$, and $\lambda$ is a coupling constant. The function $\rho$ is the normalized density function, which accounts for the coupling between the harmonic oscillator and the scalar field. Similarly to the coupling between charges and electromagnetic fields in classical electrodynamics, we shall consider in this work a point-like coupling between the harmonic oscillator and the scalar field, namely the one in which $\rho(t, \vec{x})=\delta^{(3)}(\vec{x}) / \sqrt{-g(t, \vec{x})}$, where $\delta^{(3)}$ is the three-dimensional Dirac delta function. This type of coupling requires a renormalization of the frequencies, and to this end we need an $\varepsilon$-family of uncoupled frequencies $\omega_{\varepsilon}$ (see section III]). Here and in what follows units in which $c=1$ are used.

Varying the action (2.1) with respect to $\phi$ and $Q$ one obtains the coupled evolution equations of the system, namely

$$
\begin{aligned}
& {\left[\square+\frac{1}{6} \hat{R}\right] \phi(t, \vec{x})=\lambda \rho(t, \vec{x}) Q(t),} \\
& \ddot{Q}(t)+\omega_{\varepsilon}^{2}(t) Q(t)=\lambda \int d^{3} x \sqrt{-g} \rho(t, \vec{x}) \phi(t, \vec{x}),
\end{aligned}
$$

where $\square \phi \equiv(\sqrt{-g})^{-1} \partial_{\mu}\left(\sqrt{-g} g^{\mu \nu} \partial_{\nu} \phi\right)$ is the d'Alembertian operator, and $\square+\frac{1}{6} \hat{R}$ is the wave operator defined on $\mathcal{M}_{4}$, hereafter simply called wave operator [32,33.

Before proceeding to the discussion of the Green functions for the wave operator in flat FRW space-time manifolds (for any smooth $A(t)$ ) we shall consider how one can obtain the radiation reaction equation from the above equations (2.2) and (2.3). One first solves equation (2.2) as an initial value problem, by writing the solution in the form [34] $\phi(t, \vec{x})=\phi_{I}(t, \vec{x})+\phi_{H}(t, \vec{x})$, where $\phi_{H}(t, \vec{x})$ satisfies the corresponding homogeneous equation, and where the solution of the inhomogeneous equation is given by

$$
\phi_{I}(t, \vec{x})=\int d t^{\prime} d^{3} x^{\prime} \sqrt{-g\left(t^{\prime}, \vec{x}^{\prime}\right)} \mathcal{G}\left(t, \vec{x} ; t^{\prime}, \vec{x}^{\prime}\right) \lambda \rho\left(t^{\prime}, \vec{x}^{\prime}\right) Q\left(t^{\prime}\right),
$$

with $t^{\prime} \in\left[t_{0}, t\right], \vec{x}^{\prime} \in \mathcal{M}_{3}$. In (2.4) $\mathcal{G}\left(t, \vec{x} ; t^{\prime}, \vec{x}^{\prime}\right)$ is the retarded Green function, often referred to as fundamental solution of the wave operator 33 . Since the scalar field of our system may be thought of as the propagation medium 
for the radiating energy, we assume the initial condition $\left(\phi\left(t_{0}, \vec{x}\right), \partial_{t} \phi\left(t_{0}, \vec{x}\right)\right)=(0,0)$, which means that the scalar field carries no energy at $t=t_{0}$. This condition implies that $\phi_{H}(t, \vec{x})=0$, and therefore $\phi(t, \vec{x})=\phi_{I}(t, \vec{x})$. Finally, the radiation reaction equation of the energy source $Q(t)$ can be found by using the relation $\phi=\phi[Q]$ in equation (2.3). We shall return to this point later in the next section.

In this work, the Green function plays an essential role in that it incorporates both the geometrical and topological features of the FRW $t=$ const 3 -spaces. They will be obtained, in the next section, through the study of null geodesics of the space-times for each distinct 3 -space (see table I).

\section{GREEN FUNCTIONS FOR THE WAVE OPERATOR}

In this section we shall derive the Green functions of the wave operator for the flat FRW space-times whose spacelike $t=$ const sections are the multiply connected compact orientable 3 -spaces, obtained by suitable identification of faces of a basic cell (see Refs. [2, [4]) according to the table [1, and the simply connected space $\mathcal{R}^{3}$. Clearly the metric tensor we shall be concerned is given in cartesian coordinates by

$$
g_{\mu \nu}=\operatorname{diag}\left(1,-A^{2}(t),-A^{2}(t),-A^{2}(t)\right)
$$

Regarding the manifold $\mathcal{T}_{4}$ one often encounters, in the literature on compact orientable flat 3-spaces, reference to a cube in which each pair of opposite faces is identified after half a turn 2] - [4]. However, if such a cube is endowed with the Euclidean geometry then the resulting three-space is not a manifold, but an orbifold [35, 36, 41]. This 3-space fails to be a manifold along the edges of the cube. Only when the cube is endowed with the elliptic geometry the resulting space is indeed a manifold, namely the well known real projective space $\mathcal{P}^{3}$.

Recently, using theorem 3.5.5 by Wolf [1], it has been shown that a basic cell for the Euclidean manifold $\mathcal{T}_{4}$ is the cube shown in figure 1 [41]. It should be stressed that the basic cell for $\mathcal{T}_{4}$ shown in figure 1 is a standard basic cell; in general, however, the basic cell for $\mathcal{T}_{4}$ needs not to be a cube.

\begin{tabular}{||c|c|c||}
\hline \hline Topology Type & A Basic Cell & Identifications of Faces \\
\hline \hline $\mathcal{T}_{1}$ & cube & 3 pairs non rotated \\
\hline $\mathcal{T}_{2}$ & cube & 2 pairs non rotated, 1 pair rotated $90^{\circ}$ \\
\hline $\mathcal{T}_{3}$ & cube & 2 pairs non rotated, 1 pair rotated $180^{\circ}$ \\
\hline $\mathcal{T}_{4}$ & cube & 1 pair rotated $180^{\circ}, 2$ pairs according to figure 1 \\
\hline $\mathcal{H}_{1}$ & hexagonal prism & top and bottom rotated $60^{\circ}$ \\
\hline $\mathcal{H}_{2}$ & hexagonal prism & top and bottom rotated $120^{\circ}$ \\
\hline \hline
\end{tabular}

TABLE I. The six compact orientable topologies for the flat 3-manifolds can be obtained by identifying opposite faces of a basic cell as shown in this table. 


\section{The cubes and $\mathcal{R}^{3}$ cases}

We shall discuss in this part the Green functions for the wave operator on 3-spaces $\mathcal{T}_{i}(i=1, \cdots, 4)$ shown in table [. The manifold $\mathcal{T}_{1}$, referred to in the literature as the three-torus $T^{3}$ [37], is a compact multiply connected Riemannian manifold, which can be obtained by identifying the opposite faces of a cube of side $a$.

To build the Green function in $\mathcal{R} \times \mathcal{T}_{1}$ we first use the conformal properties of FRW space-times. Defining the conformal time $\tau \equiv f(t) \equiv \int d t^{\prime} / A\left(t^{\prime}\right)$ for $t^{\prime} \in\left[t_{0}, t\right]$, we transform the problem of finding the Green function $\mathcal{G}\left(t, \vec{x} ; t^{\prime}, \vec{x}^{\prime}\right)$ of the operator $\left(\square+\frac{1}{6} \hat{R}\right)$ into the problem of finding the Green function $G\left(\tau, \vec{x} ; \tau^{\prime}=0, \vec{x}^{\prime}\right)$ of the operator $\square$ [22, 33. Moreover, a useful way of thinking about $\mathcal{T}_{1}$ in terms of the simply connected 3-manifold $\mathcal{R}^{3}$ is to imagine the cube repeated endlessly in a three-dimensional grid (basic cell and its images), where each repetition consists of the same physical region of space. The space $\mathcal{R}^{3}$ containing an infinite grid of copies of the basic cell is called the universal covering space of $\mathcal{T}_{1}$. In this way we now transform the problem of calculating the Green function of $\square$ at $(\tau, \vec{x}) \in \mathcal{R} \times \mathcal{T}_{1}$ due to a point-like source located at $\vec{x}^{\prime}=(0,0,0)$ and emitting at $\tau^{\prime}=0$, to the equivalent problem of finding the field at $(\tau, \vec{x}) \in \mathcal{R} \times \mathcal{R}^{3}$ due to an infinite set of point-like sources, each one located at the center of a cube of the grid, and all of them irradiating simultaneously at $\tau^{\prime}=0$.

In this infinite grid picture the Green function of $\square$ is calculated summing up the contributions of each one of the point-like sources. Since a scalar ray is a null geodesic, the distance travelled by each scalar wave front is a measure of the corresponding time travel, so the conformal time $\tau$ of arrival at $\vec{x}$ will depend on the position at which each source is located. Null geodesics corresponding to retarded waves connecting $\left(\tau^{\prime}=0, \vec{a}(\vec{n})\right)$ to the observation point $(\tau, \vec{x})$ are then such that $\tau-|\vec{x}-\vec{a}(\vec{n})|=0$, so the Green function for $\tau>0$ is given by

$$
G\left(\tau, \vec{x} ; \tau^{\prime}=0, \vec{x}^{\prime}=0\right)=\frac{1}{4 \pi R(\tau) R\left(\tau^{\prime}=0\right)} \sum_{n_{1}, n_{2}, n_{3}} \frac{\delta(\tau-|\vec{x}-\vec{a}(\vec{n})|)}{|\vec{x}-\vec{a}(\vec{n})|},
$$

where $\vec{x}, \vec{a}(\vec{n}) \in \mathcal{R}^{3}, \vec{a}(\vec{n}) \equiv a\left(n_{1} \vec{e}_{1}+n_{2} \vec{e}_{2}+n_{3} \vec{e}_{3}\right)$. Here $\left\{\vec{e}_{i}\right\}$ is the usual orthonormal basis vectors in $\mathcal{R}^{3}$ and $\left(n_{1}, n_{2}, n_{3}\right)$ are integers, and $R(\tau) \equiv A\left(f^{-1}(\tau)\right)$.

We shall consider now the radiation reaction equation of our energy source in $\mathcal{T}_{1}$. Using the Green function $G$ and (2.4) one finds $\phi[Q]$, which can be used in (2.3) to furnish the radiation reaction equation of our system. Note that in these calculations the first term $\left(n_{1}=n_{2}=n_{3}=0\right)$ of the Green function gives rise to the term $\left(\lambda^{2} / 4 \pi\right) \int d \chi \delta(\chi) /(A(t) \chi)$, that formally diverges. A suitable renormalization procedure is therefore necessary. This resembles the need for renormalization one finds when dealing with accelerated point charges in classical electrodynamics [30]. We learn from [28] that a renormalization procedure can always be made in this case. This amounts to saying that for any $0<\Omega^{2}<\infty$, and any $\varepsilon>0$, one can always define an $\varepsilon$-parameter family of frequencies $\omega_{\varepsilon}^{2}$ by

$$
\omega_{\varepsilon}^{2}(t) \equiv \Omega^{2}+\frac{2 \Gamma}{A(t)} \int d \chi \frac{\rho_{\varepsilon}(\chi)}{\chi}
$$

such that $\rho_{\varepsilon}(\chi)$ is a $\delta$-sequence that in the limit $\varepsilon \rightarrow 0$ converges to the Dirac delta $\delta(\chi)$.

Using now (3.3), in which we have made $2 \Gamma \equiv \lambda^{2} /(4 \pi)$, one finds that, for arbitrary initial data $\left(Q\left(t_{0}\right), \dot{Q}\left(t_{0}\right)\right)$, the renormalized radiation reaction equation of the source can be written in the form

$$
\ddot{Q}(t)+2 \Gamma \dot{Q}(t)+\Omega^{2} Q(t)=\frac{2 \Gamma}{A(t)} \sum_{n_{1}, n_{2}, n_{3}} \frac{1}{a(\vec{n})} \Theta\left(t-t_{n}\right) Q\left(f^{-1}(f(t)-a(\vec{n}))\right),
$$

for any $t \in\left[t_{0}, \infty\right)$, and where $a(\vec{n}) \equiv|\vec{a}(\vec{n})|=a\left[n_{1}{ }^{2}+n_{2}{ }^{2}+n_{3}{ }^{2}\right]^{1 / 2}$ for all $\left(n_{1}, n_{2}, n_{3}\right)$ integers not all zero, $t_{n} \equiv f^{-1}(a(\vec{n}))$ and the step distribution $\Theta(s)=0$ for any $s \leq 0, \Theta(s)=1$ for all $s>0$. Note that the radiation reaction equation (3.4) contains an infinite, but countable, number of retarded terms. Note also that, for a given pair of initial data $\left(Q\left(t_{0}\right), \dot{Q}\left(t_{0}\right)\right)$, the continuity of $Q$ and $\dot{Q}$ for all $t$ is sufficient to ensure that (3.4) can be integrated to give a unique solution.

The next 3-manifolds we shall consider are $\mathcal{T}_{2}, \mathcal{T}_{3}$ and $\mathcal{T}_{4}$ (see table $\mathbb{1}$ ). The 3 -space $\mathcal{T}_{2}$ is a compact multiply connected flat three-dimensional Riemannian manifold, obtained by identifying opposites faces of a cube, with a pair being identified after a rotation of $90^{\circ}$ of one face relative to the opposite face. The 3 -manifold $\mathcal{T}_{3}$ is obtained by identification of opposites faces of a cubic cell, but now a pair of faces are identified after a turn of $180^{\circ}$ (see table [l]). Finally, the 3-manifold $\mathcal{T}_{4}$ is obtained by pairwise identifying the faces of the cube according to figure 1 . For these 3 -spaces the Green functions for the wave operator can again be built by using the infinite grid of cubes picture. It 
should be noticed, however, that the grids of cubes corresponding to $\mathcal{T}_{1}, \mathcal{T}_{2}, \mathcal{T}_{3}$ and $\mathcal{T}_{4}$ are different . Nevertheless, as the center of a basic cell in each case represents the same infinite set of points (images of the point-like source at $(0,0,0))$ of the covering space $\mathcal{R}^{3}$, the Green functions for the wave operator in these 3 -spaces are equal to the one we have calculated for $\mathcal{T}_{1}$, i.e., are given by (3.2). Thus, for arbitrary initial data $\left(Q\left(t_{0}\right), \dot{Q}\left(t_{0}\right)\right)$ the radiation reaction equation in $\mathcal{T}_{2}, \mathcal{T}_{3}$ and $\mathcal{T}_{4}$ reduces to (3.4) obtained for $\mathcal{T}_{1}$.

A word of clarification is in order here: the above Green functions for the wave operator for the 3 -spaces $\mathcal{T}_{i}$ $(i=1, \cdots, 4)$ coincide only because in each case the point-like source is located at the center of the basic cell $\vec{x}^{\prime}=0$. This is so because although all these 3 -spaces are locally homogeneous, the 3 -spaces $\mathcal{T}_{2}, \mathcal{T}_{3}$ and $\mathcal{T}_{4}$ are not globally homogeneous, thus the corresponding Green functions of $\square$ for $\vec{x}^{\prime} \neq 0$ are more involved and do not depend simply on the relative position between the source and the observer.

Regarding the simply connected flat 3-dimensional Riemannian manifold $\mathcal{R}^{3}$ ( $t=$ const section of the Minkowski space-time), the Green function for the wave operator $\square$ is equal to the first term $\left(n_{1}=n_{2}=n_{3}=0\right)$ of $G$ appearing in (3.2). Thus the radiation reaction equation in this manifold reduces to (3.4) with right hand side equal to zero, for all $t \in\left[t_{0}, \infty\right)$.

\section{The hexagonal prism cases}

We shall discuss now the Green functions for the wave operator on the flat hexagonal prism manifolds $\mathcal{H}_{1}$ and $\mathcal{H}_{2}$ described in table I.

The 3 -space $\mathcal{H}_{1}$ is a compact multiply connected flat three-dimensional Riemannian manifold, which can be obtained by identifying the top and bottom faces of a regular hexagonal prism after a rotation of $60^{\circ}$, while the lateral faces are pairwise identified in the usual manner. The 3-manifold $\mathcal{H}_{2}$ can be similarly defined, but now the identification of the top and bottom faces (regular hexagons) is made after a turn of $120^{\circ}$. In both cases we denote by $a$ the shortest distance between two opposite sides of the regular hexagon and by $h$ the height of the hexagonal prism cell.

For a point-like source located at the center of the basic cell, the Green functions for the wave operator in the 3-manifolds $\mathcal{H}_{1}$ and $\mathcal{H}_{2}$ are obviously the same. Moreover they turn out to be similar to the Green function (3.2). But now instead of $\vec{a}(\vec{n})$ one has $\vec{b}(\vec{n}) \equiv a n_{1} \frac{\sqrt{3}}{2} \vec{e}_{1}+a\left(\frac{1}{2} n_{1}+n_{2}\right) \vec{e}_{2}+h n_{3} \vec{e}_{3}$, where $\left(n_{1}, n_{2}, n_{3}\right)$ are integers, to locate the centers of the hexagonal prism cells in which $\mathcal{R}^{3}$ has been tessellated. Note that the number of images at a given distance from the point-like source clearly depends upon the ratio $h / a$. The radiation reaction equation in the present cases is similar to (3.4) but with the obvious change $a(\vec{n}) \rightarrow b(\vec{n}) \equiv|\vec{b}(\vec{n})|=\left[a^{2}\left(n_{1}{ }^{2}+n_{1} n_{2}+n_{2}{ }^{2}\right)+h^{2} n_{3}{ }^{2}\right]^{1 / 2}$ and again the integers $\left(n_{1}, n_{2}, n_{3}\right)$ are not all zero.

It should be emphasized that as the 3 -manifolds $\mathcal{H}_{1}$ and $\mathcal{H}_{2}$ are locally but not globally homogeneous, the Green function for the wave operator in these manifolds is again given by (3.2), with $\vec{b}(\vec{n})$ instead of $\vec{a}(\vec{n})$, only because the point-like source is at the center of the basic cell $\vec{x}^{\prime}=0$.

To close this section we emphasize that the Green functions, obtained for flat FRW space-time manifolds $\mathcal{M}_{4}=$ $\mathcal{R} \times \mathcal{M}_{3}$, contain the topological constraints of $\mathcal{M}_{3}$, information that makes possible to find out the exact radiation reaction equation in each case.

\section{NUMERICAL ANALYSIS}

In this section we shall discuss the time behavior of the energy of the harmonic oscillator $E(t)=\frac{1}{2}\left[\dot{Q}^{2}(t)+\Omega^{2} Q^{2}(t)\right]$, where the function $Q(t)$ is the solution of the radiation reaction equation corresponding to each flat manifold we have discussed in the previous section.

Without loss of generality, in the integration of the radiation reaction equations and in the plotting of the energy function we have taken specific values for the constants. We have also assumed the continuity of $Q$ and $\dot{Q}$, and chosen suitable values for the initial data $Q\left(t_{0}\right)$ and $\dot{Q}\left(t_{0}\right)$. For a neat comparison between the simply and multiply connected cases we have chosen $\Gamma=1, \Omega^{2}=30$, the length $a=1$, and for the heights of the hexagonal prism and three-torus we have taken $h=0.4$ and 1 . As a matter of fact, our three-torus $\mathcal{T}_{1}$ was obtained from a parallelepiped with edges $a, a$, $h$, so $a(\vec{n})=\left[a^{2}\left(n_{1}^{2}+n_{2}^{2}\right)+h^{2} n_{3}^{2}\right]^{1 / 2}$ was used in equation (3.4). Further, we have also chosen as initial data $t_{0}=0,(Q(0), \dot{Q}(0))=(\sqrt{2} / \Omega, 0)$, which means that the initial energy of the source has been normalized, i.e., $E(0)=1$.

\footnotetext{
${ }^{1}$ This is so because each 3-manifold is obtained by forming the quotient $\mathcal{M}_{3}=\mathcal{R}^{3} / \Gamma_{i}(i=1, \cdots, 4)$, where for each case $\Gamma_{i}$ is a different discrete group of isometries of the covering space $\mathcal{R}^{3}$ acting properly discontinuously, without fixed points [2].
} 
Figures 2, 3 and 4 correspond to the static case $A(t)=$ const $\equiv A_{0}$, which we have normalized to $1\left(A_{0}=1\right)$, while dynamic situations $(\dot{A}(t) \neq 0)$ are considered in figure 5 .

Taking into account the above choices of values and using the computer algebra systems Mathematica [38] and Maple [39] the numerical integrations of the radiation reaction equations as well as the corresponding graphs for the energy function were obtained (see figures 2 to 5 ).

Figure 2 shows the behavior of the energy with the time for the flat FRW space-times, in which the $t=$ const sections are any of the orientable compact multiply connected 3-manifolds $\mathcal{T}_{1}, \mathcal{T}_{2}$ and $\mathcal{T}_{3}$ and $\mathcal{T}_{4}$ for different ratios $h / a$. Note that $h$ is the height of the parallelepiped of square basis of side $a$. The curves exhibit basically the same pattern, namely relative minima and maxima followed by a predominant growth of the energy with the time $(E(t) \rightarrow \infty$ when $t \rightarrow \infty)$. These extrema are related to the contribution of the discrete retarded terms (the right hand side of the radiation reaction equation) demanded by the compactness and the corresponding connectedness of these manifolds. The fact that the relative extrema occur at different times and are of different amplitudes (intensities) for distinct tessellations (different ratios $h / a$ ) of the covering manifold, basically reveals the differences in their degree of connectedness (returning rays take different times to return to the origin). This growth of the energy is discussed in the next section.

Regarding the behavior of the energy function for the cases in which the $t=$ const sections are $\mathcal{H}_{1}$ and $\mathcal{H}_{2}$, shown in figure 3 , we again note that due to the compactness and connectedness we have relative minima and maxima with distinct intensities, which take place at different instants for distinct ratios $h / a$. The curves again display an eventual growth of the energy with the time for these manifolds.

Figure 4 compares the variation of the energy $E$ with the time for the cases where the $t=$ const section $\mathcal{M}_{3}$ are $\mathcal{R}^{3}, \mathcal{T}_{1}$ (with $a=h=1$ ) and $\mathcal{H}_{1}$ (also with $a=h=1$ ). This figure shows for the Minkowski space-time, as expected, an exponential decay of the energy with the time, whereas for the manifolds $\mathcal{T}_{1}$ and $\mathcal{H}_{1}$ it shows basically the same pattern, i.e. relative minima and maxima occurring at different times, depending on the degree of connectedness, followed by a growth of the energy with the time.

Although the net role played by the degree of connectedness as well as compactness can be singled out in the static cases $A(t)=$ const $\equiv A_{0}$, for the sake of completeness we have examined three instances where dynamic expansion takes place. Figure 5 corresponds to the plot of the energy function for the $\mathcal{T}_{1}$ manifold (with $a=h=1$ ) in the dynamic expanding cases: (i) linear expansion $A(t)=t+0.7$, (ii) square root expansion $A(t)=\sqrt{t}$, (iii) inflationary expansion $A(t)=e^{t / \sqrt{2}}$, that is, an expansion with future event horizon.

Although for the case (iii) one clearly has radiation damping, we have not been able to find out so far a closed formal proof of the asymptotical behavior for the other two cases. We emphasize, nevertheless, that the net role played by the connectedness and compactness can be better singled out in the static cases, where the dynamical degrees of freedom are frozen. The study of that role for the static cases is in fact the major aim of the present work.

\section{CONCLUSIONS AND FINAL REMARKS}

In this work we have studied the role played by the topological compactness and connectedness in the time evolution of the energy of an harmonic oscillator in flat FRW space-time manifolds, whose $t=$ const sections are (i) the orientable simply connected non-compact 3 -space $\mathcal{R}^{3}$ and (ii) six possible flat orientable multiply connected compact 3-manifolds given in table [.

For the $\mathcal{R}^{3}$ case we found that the energy function $E(t)$ exhibits an exponential decay with the time - the radiation damping $(E(t) \rightarrow 0$ when $t \rightarrow \infty)$ takes place, as one could have expected in agreement with [20] - [26].

For the manifolds $\mathcal{T}_{1}, \mathcal{T}_{2}, \mathcal{T}_{3}$ and $\mathcal{T}_{4}$ as well as for the manifolds $\mathcal{H}_{1}$ and $\mathcal{H}_{2}$ the behavior of the energy with the time exhibits basically the same pattern: relative minima and maxima occur at different times for distinct ratios $h / a$ (distinct tessellations) depending on the degree of connectedness of each 3-manifold, and are followed by a growth of $E(t)$.

This asymptotical divergent behavior of $E(t)$ for these compact manifolds contrasts with the radiation damping of the energy we have found for $\mathcal{R}^{3}$. There is a quite simple heuristic argument which suports our numerical results, though. If one ad hoc assumes an exponential asymptotical behavior for $Q$, i.e. $Q(t)=\gamma \exp (\beta t)$ with $\beta$ and $\gamma$ real constants, then for the static cases $(A(t)=1)$, and for each of the above compact manifolds, in the limit $t \rightarrow \infty$ equation (3.4) reduces to

$$
\frac{\beta^{2}+2 \Gamma \beta+\Omega^{2}}{2 \Gamma}=\sum_{m=1}^{\infty} \frac{c_{m}}{a_{m}} \exp \left(-\beta a_{m}\right),
$$

where $c_{m}$ is the number of images of the point-like source at a distance $a_{m}$ in the infinite grid picture. To attain our 
goal, we will show that (5.1) has only one real solution for $\beta$, which is positive. Indeed, let $f(\beta)$ be the right hand side of (5.1), which is a positive monotone decreasing function of $\beta$, and such that

$$
\lim _{\beta \rightarrow 0} f(\beta)=\infty \quad \text { and } \quad \lim _{\beta \rightarrow \infty} f(\beta)=0 .
$$

Thus $f(\beta)$ lies entirely in the first quadrant of the plane and crosses it from the top-left to the bottom-right. Now, since $\Gamma>0$ then for a given pair $(\Gamma, \Omega)$ the left hand side of (5.1) is a parabola curved upwards with vertex at $\beta=-\Gamma$. Therefore, it always intersects the curve for $f(\beta)$ in just one point, which is in the first quadrant. In other words, there is only one real $\beta$ solution to equation (5.1), which is positive.

The unexpected (unphysical ?) growth of the energy with the time for the above compact flat 3-manifolds cases illustrates that non-trivial topologies can induce rather important dynamic changes in the behavior of a physical system. This type of sensitivity has been refered to as topological fragility and can occur without violation of any local physical law [18]. A rigorous non-numerical analysis of the reasons for this surprising divergent behavior of $E(t)$ when compact flat FRW space-times are considered has been carried out, and we hope to publish our results shortly elsewhere. We anticipate, however, that the causes for such a behavior lie in the compactness of the manifold in at least one direction, on the one hand, and in the type of coupling between $Q$ and $\phi$, on the other hand.

A possible physical measure of the degree of connectedness in these compact 3-manifolds can be made through the study of the number of emitted rays that return to the origin within a given lapse of time. According to this concept of degree of connectedness one learns from figures 2, 3 and 4 that the greater is the degree of connectedness the earlier is the occurrence of the first relative minimum in the energy function. Incidentally, note that the extension of this concept of degree of connectedness to non-compact 3-manifolds implies that $\mathcal{R}^{3}$ has a null degree of connectedness. This, of course, is indicated in figure 4, which shows a net exponential decay of the energy with the time; no relative minima and maxima come about, which means that no ray returns to the origin.

A simple inspection of the graphs for $E(t)$ clearly shows that the derivative $\dot{E}=\dot{Q}\left(\ddot{Q}+\Omega^{2} Q\right)$ of the energy function is discontinuous at a few points. Indeed, for the static case, for example, using (3.4) one obtains

$$
\dot{E}(t)=2 \Gamma \dot{Q}(t)\left[\sum_{n_{1}, n_{2}, n_{3}} \frac{1}{a(\vec{n})} \Theta(t-a(\vec{n})) Q(t-a(\vec{n}))-\dot{Q}(t)\right] .
$$

From this equation one sees that the discontinuities occur at $t=a(\vec{n})$, that is, they come about each time a new term $Q(t-a(\vec{n})) / a(\vec{n})$ is taken into account in the right hand side of (3.4). A question which naturally arises here is whether the inverse problem, i.e. that of determining the basic cell (topology) corresponding to the spacelike $t=$ const sections from the graphs of the energy $E(t)$, can be solved. Regarding this problem it is clear that one can find the distances of the point-like source to its images by using where the discontinuities of $\dot{E}(t)$ take place, and the number of images at a given distance through the magnitude of the corresponding discontinuities. So, one can probe the topology of the 3-spaces at least in a few cases. It is not yet clear whether an algorithm for solving the inverse problem for the most general (Euclidean) setting can be found, though.

As far as we are aware [3] this is the first work in which a physical consequence of adopting the flat hexagonal prisms $\mathcal{H}_{1}$ and $\mathcal{H}_{2}$ has been studied.

It is of worth to emphasize that when the expansion of the universe is considered the degree of connectedness is less than the ones for the static cases. For the manifold $\mathcal{T}_{1}$ this can be seen by comparing figure 2 (static case $\dot{A}(t)=0$ ) and figure 5 (monotone expansions $\dot{A}(t)>0$ ).

Before closing this article we should like to stress that our study does not cover all possible spatially compact orientable flat FRW manifolds. Thus, for example, we have not considered that for the 3-manifolds $\mathcal{T}_{1}$ and $\mathcal{T}_{3}$ the basis of the basic cell need not to be a square, it can be a parallelogram. The restriction we have made, however, does not seem to be decisive for the patterns of the behavior of the energy with the time we have found.

To conclude we remark that the study of radiation damping in elliptic $(\kappa=1)$ FRW manifolds in which the $t=$ const sections are endowed with different (orientable compact) topologies is being carried out.

\section{ACKNOWLEDGEMENTS}

We are grateful to an anonymous referee for valuable suggestions. We also thank the Brazilian scientific agencies CNPq and CAPES for financial support. A.B. is also grateful to CLAF. 
* Permanent address: Facultad de Ciencias, Universidad Nacional de Ingeniería, Apartado 31 - 139 , Lima 31 - Peru. INTERNET: bernui@fc-uni.edu.pe

$\S \quad$ INTERNET: german@cat.cbpf.br

$\dagger \quad$ INTERNET: reboucas@cat.cbpf.br

¥ INTERNET: teixeira@novell.cat.cbpf.br

[1] J.A. Wolf, Spaces of Constant Curvature, McGraw-Hill, New York (1967).

[2] G.F.R. Ellis, Gen. Rel. Grav. 2, 7 (1971).

[3] M. Lachièze-Rey \& J.-P. Luminet, Phys. Rep. 254, 135 (1995).

[4] G.F.R. Ellis \& G. Schreiber, Phys. Lett. A 115, 97 (1986).

[5] W. Oliveira, M.J. Rebouças \& A.F.F. Teixeira, Phys. Lett. A 188, 125 (1994).

[6] J.R. Gott, Mon. Not. R. Astr. Soc. 193, 153 (1980).

[7] M. Demiánski \& M. Lapucha, Mon. Not. R. Astr. Soc. 224, 527 (1987).

[8] L.Z. Fang \& H. Sato, Gen. Rel. Grav. 17, 1117 (1985).

[9] T.J. Broadhurst, R.S. Ellis, D.C. Koo \& A.S. Szalay, Nature 343, 726 (1990).

[10] H.V. Fagundes, Phys. Rev. Lett. 70, 1579 (1993).

[11] D. Stevens, D. Scott \& J. Silk, Phys. Rev. Lett. 71, 20 (1993).

[12] A.A. Starobinsky, JETP Lett. 57, 622 (1993).

[13] G.F.R. Ellis \& R.K. Tavakol, Class. Quantum Grav. 11, 675 (1994).

[14] Y.P. Jing \& L.Z. Fang, Phys. Rev. Lett. 731882 (1994).

[15] A. de Oliveira-Costa \& G. Smoot, Astrophys. J. 448, 447 (1995).

[16] N.J. Cornish, D.N. Spergel \& G.D. Starkman, "Circles in the Sky: Finding Topology with Microwave Background Radiation", gr-qc 960239 (1996).

[17] H.V. Fagundes, "Fitting hyperbolic universes to Cayón-Smoot spots in COBE's maps", Institute for Theoretical Physics preprint IFT-P.050/95 (UNESP), São Paulo (1996).

[18] M.J. Rebouças, R.K. Tavakol \& A.F.F. Teixeira, "Topology and Fragility in Cosmology", to appear in Gen. Rel. Grav. (1998).

[19] B.F. Roukema, Mon. Not. R. Astr. Soc. 283, 1147 (1996).

[20] F. Schwalb \& W. Thirring, Ergeb. exakten Naturwiss. 36, 219 (1964).

[21] W. Burke, J. Math. Phys. 12, 401 (1971).

[22] P. Aichelburg \& R. Beig, Phys. Rev. D 15, 389 (1977).

[23] W. Unruh, in Gravitational Radiation, Les Houches 1982, Eds. N. DeRuelle \& T. Piran, North-Holland, Amsterdam (1983).

[24] J. Stewart, Gen. Rel. Grav. 15, 425 (1983).

[25] J.L. Anderson, Gen. Rel. Grav. 16, 595 (1984).

[26] C. Hoenselaers \& B. Schmidt, Class. Quantum Grav. 6, 867 (1989).

[27] A. Bernui, Appl. Anal. 42, 157 (1991).

[28] A. Bernui, Ann. Physik 3, 408 (1994).

[29] R. Beig, J. Math. Phys. 19, 1104 (1978).

[30] J.D. Jackson, Classical Electrodynamics, John Wiley, New York (1975).

[31] J.B. Marion, Classical Electromagnetic Radiation, Academic Press, New York (1965).

[32] S. Sonego \& V. Faraoni, Class. Quantum Grav. 10, 1185 (1993).

[33] F.G. Friedlander, The Wave Equation on a Curved Spacetime, Cambridge U. P., Cambridge (1975).

[34] See, for example, P.M. Morse \& H. Feshbach, Methods of Theoretical Physics, Mc Graw-Hill, New York (1953).

[35] W.P. Thurston, "The Geometry and Topology of Three-Manifolds", Princeton Lecture Notes, Princeton (1979).

[36] W.P. Thurston, Bull. Amer. Math. Soc. 6, 357 (1982).

[37] J.R. Weeks, The Shape of Space, "Pure and Applied Mathematics" Vol. 26, Marcel Dekker Inc., New York (1985).

[38] S. Wolfram, Mathematica: a System for Doing Mathematics by Computer, Addison-Wesley, New York (1991).

[39] B.W. Char, K.O. Geddes, G.H. Gonnet, B.L. Leong, M.B. Monagan \& S.M. Watt, Maple V Language Reference Manual, Springer-Verlag, New York (1992).

[40] R. Lehoucq, M. Lachièze-Rey, J.-P. Luminet, Astron. \& Astrophys. 313, 339 (1996).

[41] G.I. Gomero, Fundamental Polyhedron for the Sixth Euclidean, Compact, Orientable Three-manifold, CBPF-NF-049/97, Centro Brasileiro de Pesquisas Físicas report (1997).

FIG. 1. A basic cell for the manifold $\mathcal{T}_{4}$ is a cube whose faces are pairwise identified as indicated in this figure.

FIG. 2. Behavior of the energy of the harmonic oscillator for $\Gamma=1$ and $\Omega^{2}=30$ in static flat FRW space-times with $\mathcal{T}_{1}$ space slices. There are a few relative maxima followed by a growth of $E(t)$. Two different ratios $h / a$ are considered $(h$ is the height and $a$ is the side of the square basis of the basic cell). 
FIG. 3. Behavior of the energy $E(t)$ of the harmonic oscillator for $\Gamma=1$ and $\Omega^{2}=30$ in static flat FRW space-times with 3 -space $\mathcal{H}_{1}$. There are a few relative maxima followed by a growth of $E(t)$. It shows the energy vs. time curves for distinct ratios $h / a$ ( $h$ is the height of the hexagonal prism and $a$ is shortest distance between two opposite sides of the regular hexagon).

FIG. 4. The time evolution of the energy of the harmonic oscillator for flat, static FRW space-times with different topologies for the spacelike $t=$ const sections: $\mathcal{R}^{3}, \mathcal{T}_{1}$ and $\mathcal{H}_{1}$ (both with $a=h=1$ ). Different degree of connectedness implies different patterns of $E(t)$. Here again $\Gamma=1$ and $\Omega^{2}=30$.

FIG. 5. The time evolution of the energy of the harmonic oscillator in FRW expanding space-times with 3-space $\mathcal{T}_{1}$ (for $a=h=1)$. Three types of dynamic expansion are shown: linear $[\dot{A}(t)=1]$, square root $\left[A(t)=t^{1 / 2}\right]$ and inflationary $\left[\dot{A}(t)=\alpha e^{\alpha t}, \alpha^{-1}=2^{1 / 2}\right]$. Here again $\Gamma=1$ and $\Omega^{2}=30$. 


$$
{ }^{1} F^{J} G
$$




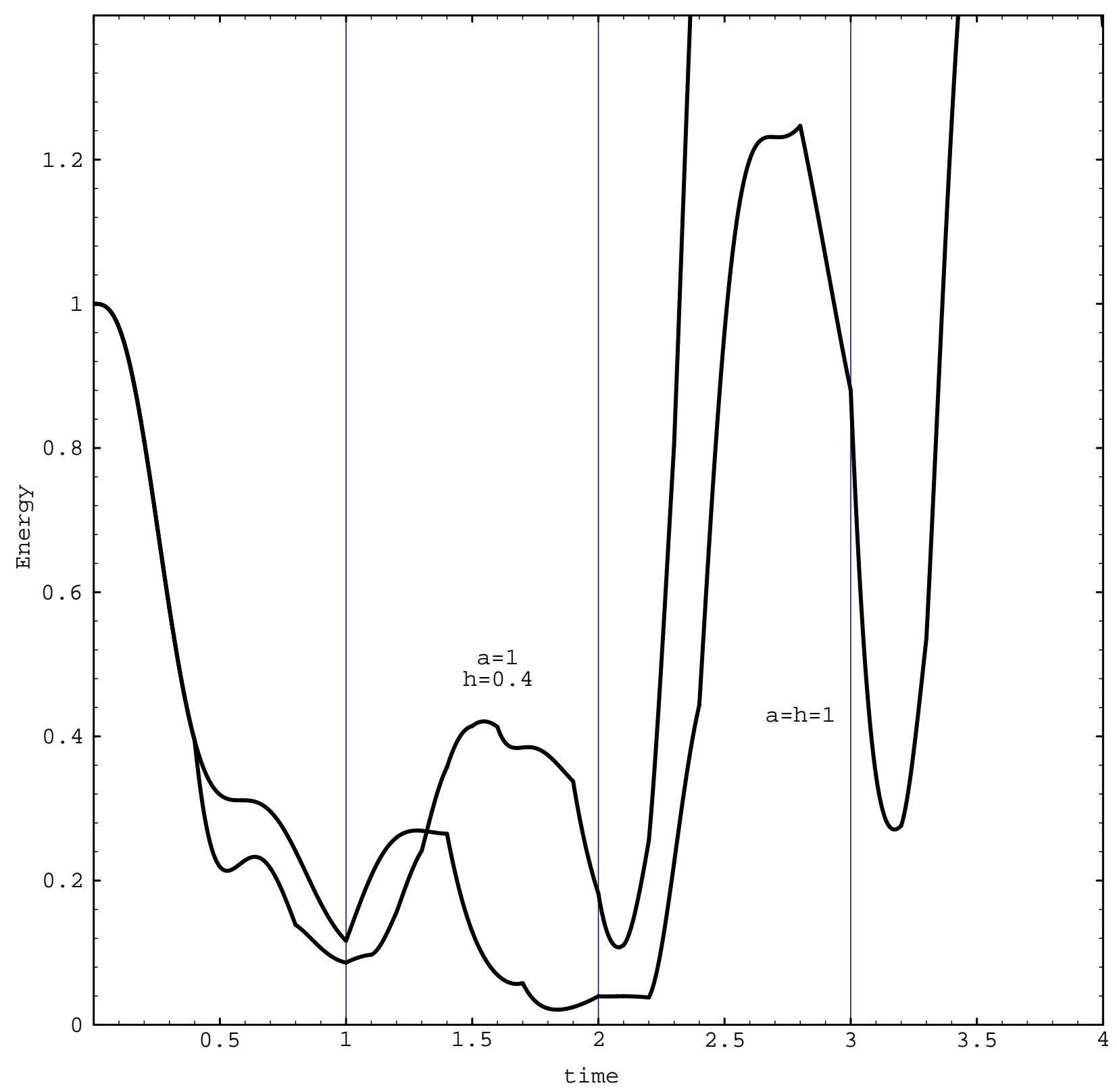




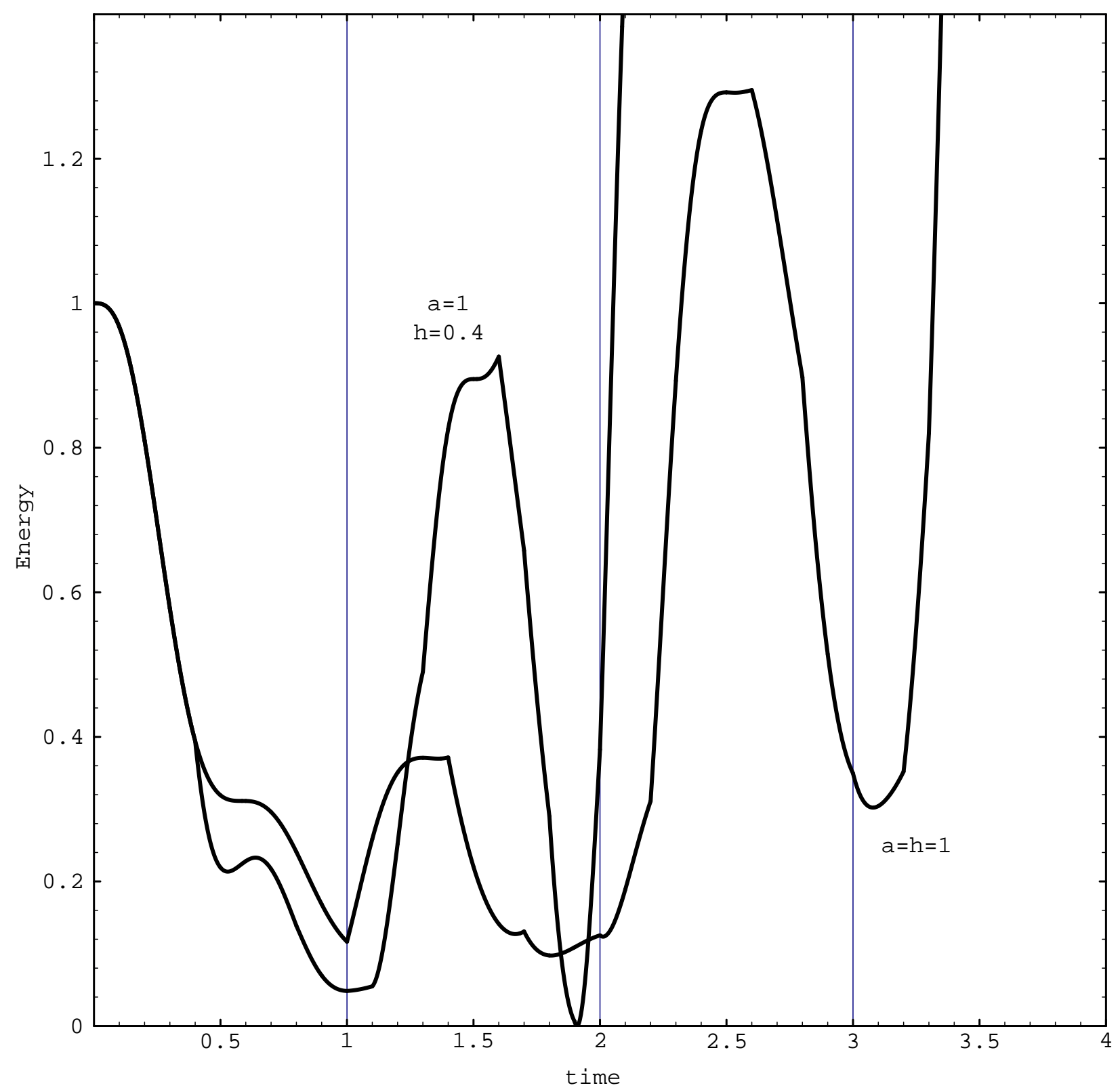




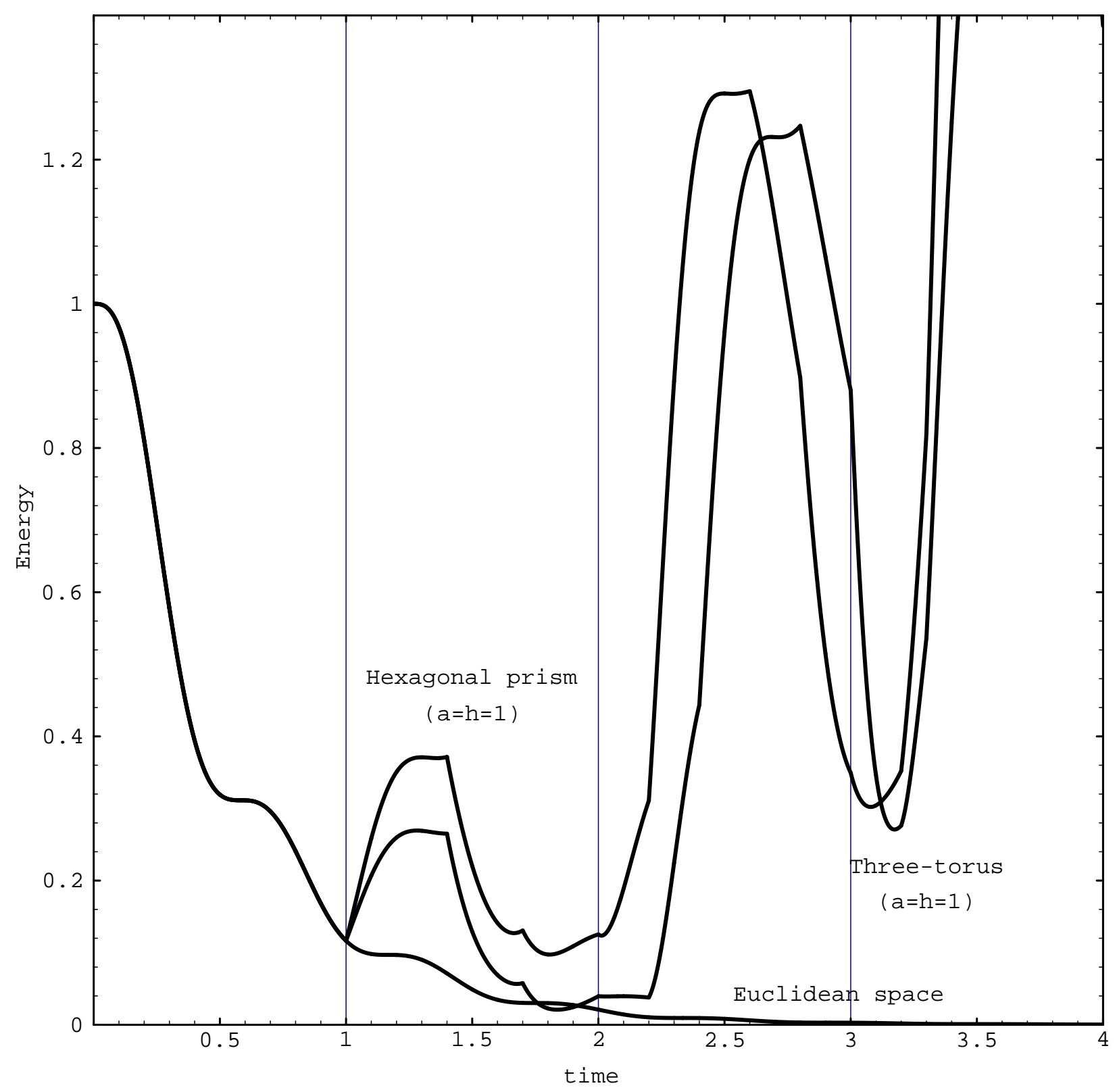




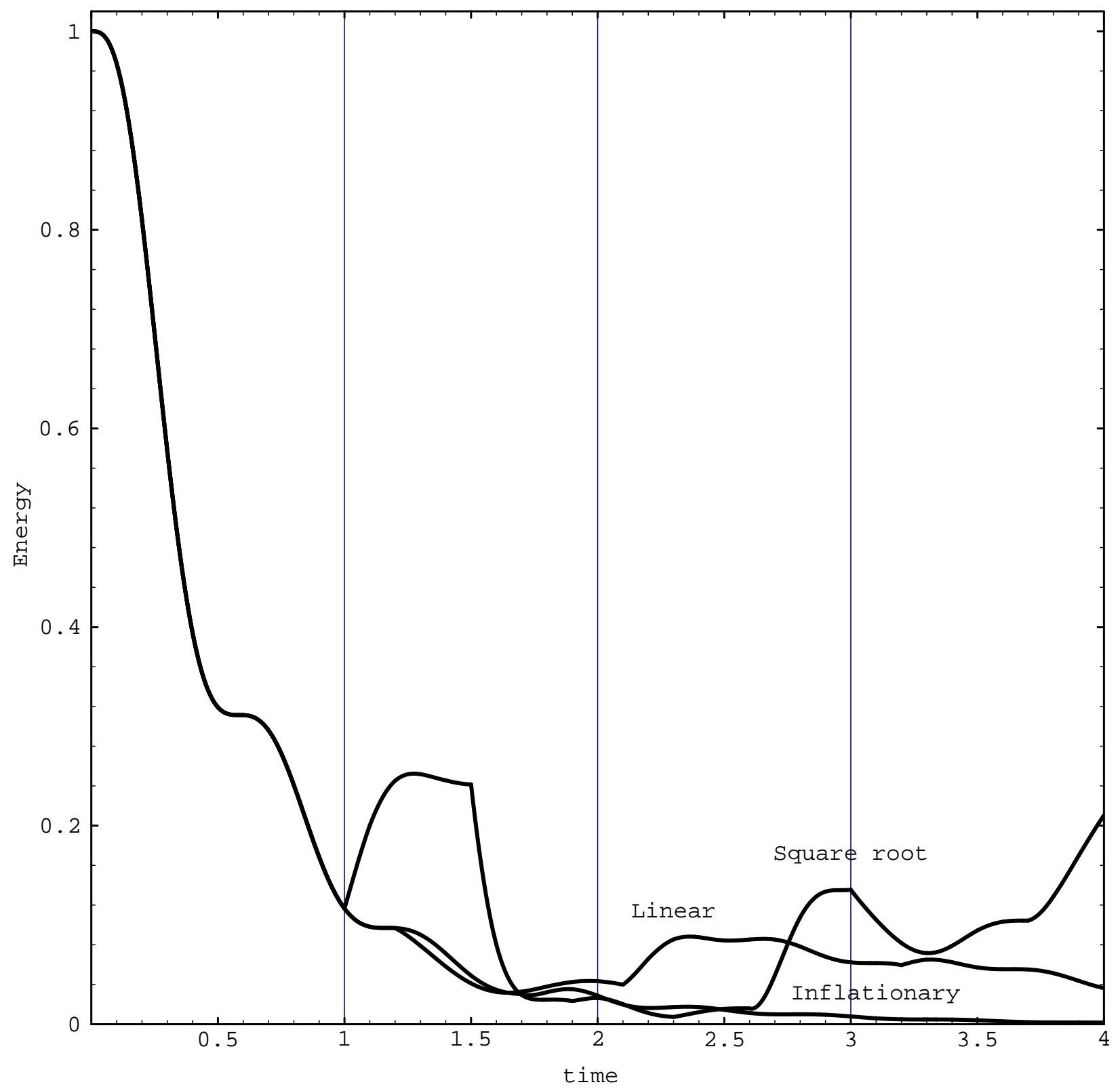

